\title{
Indices d'exploitation de ressources marines à la fin du 2nd millénaire av. J.-C. : le foyer de l'âge du bronze aux Batardières (Saint-Vincent-sur-Jard, Vendée)
}

Indices of marine resources at the end of the 2 nd millennium $B C$ : the fireplace of the bronze age to Batardières (Saint-Vincent-sur-Jard, Vendée)

Índices de explotación de los recursos marinos a finales del segundo milenio antes de Cristo: el hogar de la Edad de Bronce en Batardières (Saint-Vincent-surJard, Vendée)

Hinweise auf die Ausbeutung mariner Ressourcen am Ende des 2. Jahrtausends v. Chr.: die bronzezeitliche Feuerstelle bei Batardières (Saint-Vincent-sur-Jard, Vendée, Frankreich)

Jean-Marc Large, Sophie Corson, Catherine Dupont et Olivier Weller

\section{OpenEdition}

\section{Journals}

Édition électronique

URL : http://journals.openedition.org/rao/2951

DOI : $10.4000 /$ rao. 2951

ISBN : $978-2-7535-5014-8$

ISSN : 1775-3732

Éditeur

Presses universitaires de Rennes

\section{Édition imprimée}

Date de publication : 31 décembre 2015

Pagination : $97-112$

ISBN : 978-2-7535-5012-4

ISSN : 0767-709X

Référence électronique

Jean-Marc Large, Sophie Corson, Catherine Dupont et Olivier Weller, « Indices d'exploitation de ressources marines à la fin du 2nd millénaire av. J.-C. : le foyer de l'âge du bronze aux Batardières (Saint-Vincent-sur-Jard, Vendée) », Revue archéologique de l'Ouest [En ligne], 32 | 2015, mis en ligne le 28 avril 2018, consulté le 02 mars 2021. URL : http://journals.openedition.org/rao/2951 ; DOI : https:// doi.org/10.4000/rao.2951 


\title{
Indices d'exploitation de ressources marines à la fin du $2^{\text {nd }}$ millénaire av. J.-C. : le foyer de l'Âge du Bronze aux Batardières (Saint-Vincent-sur-Jard, Vendée)
}

\author{
Indices of Marine Resources at the End of the $2^{\text {nd }}$ Millennium BC: \\ The Fireplace of the Bronze Age to Batardières (Saint-Vincent-sur-Jard, Vendée)
}

\author{
Jean-Marc Large ${ }^{\mathrm{a}}$, Sophie Corson ${ }^{\mathrm{b}}$, Catherine Dupont ${ }^{\mathrm{c}}$ et Olivier Weller ${ }^{\mathrm{d}}$
}

\begin{abstract}
Résumé : Lors de prospections pédestres répétées, suite à la tempête Xynthia, deux petits locus archéologiques sont apparus en haut de falaise à Saint-Vincent-sur-Jard (Vendée). Si leur emprise n'est pas importante, leur localisation en front de falaise les rend sensibles à la destruction par des événements météorologiques ou bien par l'attaque de la mer lors des plus hautes marées. Ils ne craignent pas, par contre, le piétinement car le passage des promeneurs est bien canalisé par un grillage de protection qui les sépare des vestiges. L'un des locus concerne les restes d'un foyer contenant des éléments probablement d'un même vase. Les tessons qui le composent ont deux particularités : la première indique qu'ils ont été chauffés dans la structure de combustion, la seconde, par leur aspect vacuolaire, permettait d'envisager l'hypothèse d'un récipient de chauffe de saumure afin d'obtenir un pain de sel, hypothèse confirmée par une petite série d'analyses chimiques. À la périphérie du foyer, un lot de coquillages indique aussi une fonction culinaire. Enfin, deux autres restes céramiques ont été retrouvés à proximité du foyer. L'ensemble est daté de la fin du second millénaire av. J.-C.
\end{abstract}

\begin{abstract}
Two small archaeological loci were discovered at the top of cliff at Saint-Vincent-sur-Jard (Vendee) during field walks conducted after the Xynthia storm. Even though they are relatively small, their cliff-front location makes them susceptible erosion by weather or by the sea at the high tide. They are however protected from trampling by walkers by a fence. One locus concerns the remains of a hearth probably containing potsherds from the same vessel. The sherds shall have two particular features: firstly, they were heated in the hearth, secondly their vacuolar appearance, leads to the hypothesis of a brine heating vessel for obtaining salt. This hypothesis is confirmed by chemical analyzes. Shells found near to the hearth indicate that it was used for cooking. Two other vessels were also found near the hearth. The set is dated to the end of the second millenium $B C$.
\end{abstract}

Mots clés : Âge du bronze, littoral, foyer, poteries, coquillages, côte atlantique, Vendée.

Keywords: Bronze Age, coastline, hearth, pottery, shells, Atlantic coast, Vendée.

\footnotetext{
a Archéologue, chercheur associé à l'UMR 6566 CReAAH, Rennes, (jlarge2@wanadoo.fr)

${ }^{\mathrm{b}}$ Archéologue, responsable des collections archéologiques à l'Historial de la Vendée. (sophie.corson@vendee.fr)

${ }^{\mathrm{c}}$ Chargée de recherches au CNRS, UMR 6566 CReAAH, Rennes. (catherine.dupont@univ-rennes1.fr)

' Chargé de recherches au CNRS, Université Paris 1 Panthéon-Sorbonne, UMR 8215 Trajectoires, Nanterre. (olivier.weller@libertysurf.fr)
} 
Suite à la tempête Xynthia de février 2010, après la dégradation de la falaise essentiellement sablo-limoneuse sur ce trait de côte, deux petits locus archéologiques ont été repérés par Serge Deyres en haut de la falaise entre Bélesbat à Saint-Vincent-sur-Jard et la plage de Boisvinet à Jard-surMer, au lieu-dit les Batardières, en limite de commune de Saint-Vincent-sur-Jard (fig. 1). La falaise est composée d'un substrat calcaire surmonté d'un épais dépôt limono-sableux, formation périglaciaire, signature d'une phase sèche et froide du cycle périglaciaire würmien et considérée comme des nappes de solifluxion (Ters, 1961). Ce dernier se décompose en deux ensembles visibles : un limon sableux de couleur jaune à ocre posé sur le substrat calcaire et épais d'environ un mètre et un limon sableux brun à coulées mangano-ferriques positionné sur le premier et épais lui aussi d'un mètre environ. C'est sur le toit de ces niveaux que sont disposés les deux restes de structures de combustion. Au-dessus, s'est déposé le sable dunaire qui forme la couverture actuelle de la falaise.

Lors de la tempête Xynthia, deux phénomènes se sont ajoutés. Le premier consiste dans le recul du manteau dunaire sommital et/ou son effondrement sur la plage actuelle; le second a provoqué l'effondrement partiel de la falaise. Ces deux phénomènes ont libéré des espaces qui n'étaient pas visibles jusqu'alors. Lérosion plus rapide de la couche sableuse a provoqué un décrochage, formant un ressaut à paroi légèrement oblique sur une terrasse très étroite. C'est ainsi que plusieurs vestiges archéologiques sont apparus. Ils sont situés généralement entre le sommet des niveaux limono-sableux et le sable de la dune.

Sur une terrasse actuelle placée au sommet des limons, deux pointements archéologiques sont visibles. Le premier consiste en un ensemble de pierres ayant subi une rubéfaction formant une petite structure de $60 \mathrm{~cm}$ environ en haut de falaise, positionnée sur le toit des limons (fig. 2). L'un des blocs est un fragment de meule dormante en granite régional. Le second est une lentille de sédiments rubéfiés avec plusieurs éléments céramiques, d'une longueur d'environ $60 \mathrm{~cm}$ (fig. 3). Quelques coquillages s'ajoutent au matériel archéologique qui s'étend sur une longueur de deux mètres environ.

L'intervention la plus urgente a consisté à récupérer et à relever les éléments archéologiques associés à la lentille de combustion de la deuxième structure repérée (Batardières 2). La première structure n'est pas soumise à la même situation de destruction. La mise en œuvre d'une opération de terrain légère a permis de nettoyer une zone de trois mètres le long de la falaise, sur un mètre en direction du nord, c'est-à-dire sur le haut de la terrasse limoneuse actuelle. La profondeur de l'intervention est réglée par celle de la lentille rubéfiée, sur une dizaine de centimètres de hauteur. Ce nettoyage était accompagné d'un brossage de la coupe de falaise, d'un relevé en plan des éléments archéologiques et d'un prélèvement de ces mêmes éléments (céramiques, coquillages, os). Une attention particulière a été réservée aux charbons de bois afin d'obtenir une datation radiocarbone.

L'intervention archéologique a eu lieu les 4 et 5 juin 2012 et a été effectuée par des moyens manuels (décapage d'une zone de trois mètres $(\mathrm{E} / \mathrm{O})$ sur un mètre environ $(\mathrm{N} / \mathrm{S})$ et fouille par passes successives de la zone de combustion d'une longueur d'un mètre $(E / O)$ pour une largeur de $80 \mathrm{~cm}$ (N/S). C'est cette dernière zone qui contenait le plus grand ensemble de tessons de céramique. Un deuxième ensemble plus réduit était situé à environ 1,20 m à l'ouest de la lentille de combustion.

\section{LA LENTILLE DE COMBUSTION DE BÂTARDIÈRES 2}

Disposé dans une dépression de faible profondeur dans les limons bruns supérieurs, un encroûtement formé de sable et de graviers calcaires rubéfiés formait une calotte ovale d'environ $80 \mathrm{~cm}$ dans un axe est/ouest et de $50 \mathrm{~cm}$ dans un axe nord-sud. Son épaisseur maximum est d'une quinzaine de centimètres. Cet encroûtement contenait aussi quelques coquillages, essentiellement des patelles, qui ont subi aussi une combustion. À la périphérie nord de cet encroûtement (le côté sud a disparu suite à l'érosion de la falaise), un niveau coquillier d'assez faible contenance n'a pas subi la même intensité de combustion. Les restes d'au moins un vase dans un état de dégradation avancée, les tessons ayant subi aussi l'épreuve du feu, étaient disposés dans la lentille de combustion. Leur surface est très dégradée et montre des vacuoles permettant d'envisager l'hypothèse d'un contenant à sel. Quatre passes successives ont été effectuées pour permettre l'étude la plus fine de cette lentille (fig. 4 et 7). Les tessons de la céramique se trouvent dans les quatre passes, certains sont en position verticale. La similitude de cette structure avec celle de Couture dans le Marais de Rochefort, datée du Néolithique récent, implique la nécessité d'aller plus loin dans les recherches sur le lien avec une exploitation du sel entre le Néolithique récent et la Protohistoire (Ard et Weller, 2012, citant une fouille préventive menée par A. Bolle - Inrap). L'un des deux éléments de céramiques trouvés à un mètre à l'ouest de la lentille possède un départ de préhension, un autre élément possède une cannelure décorative (fig. 5). 

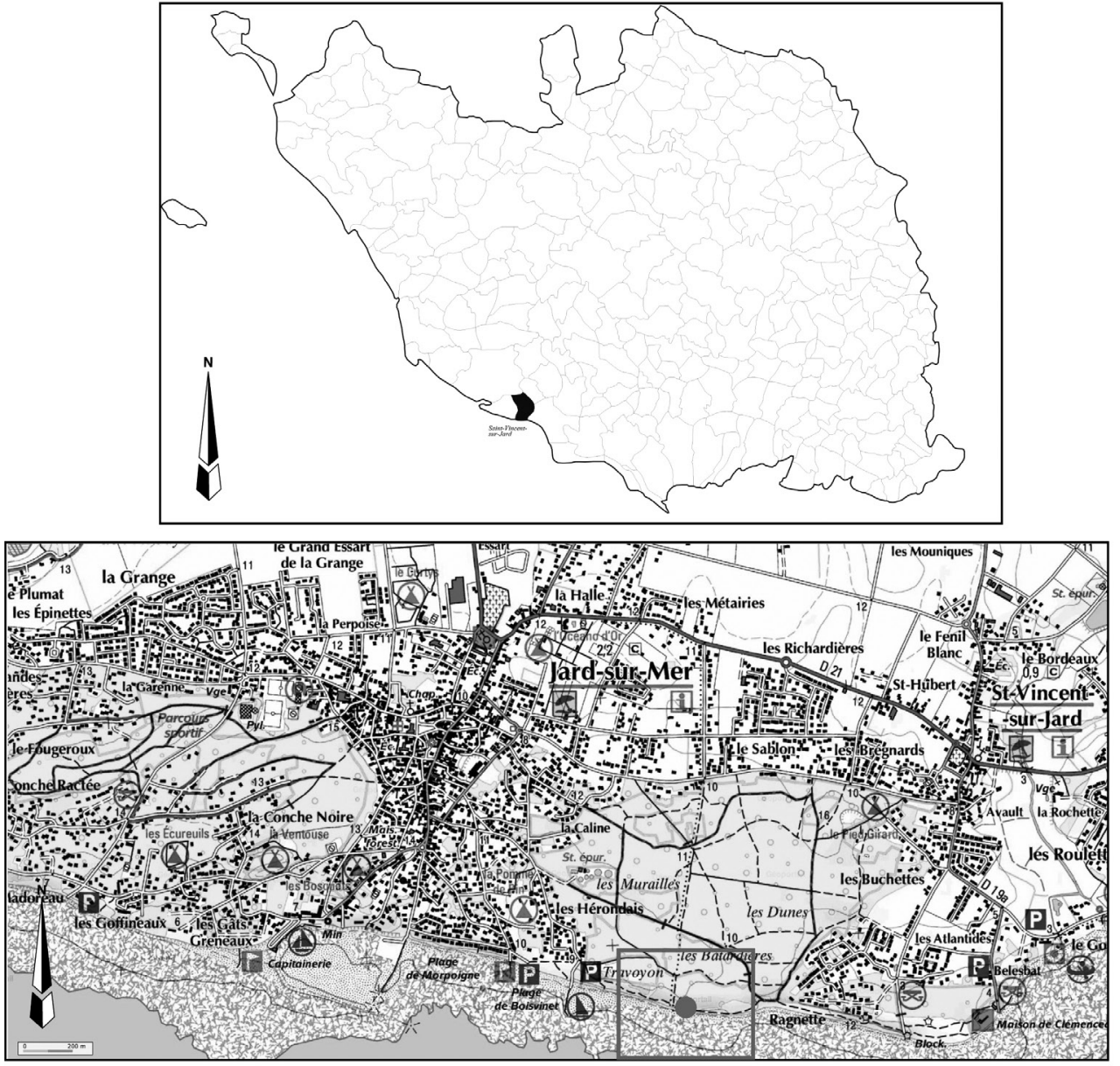

Figure 1 : Saint-Vincent-surJard (Vendée) - Situation du site de Batardières.

Figure 1: Saint-Vincent-surJard (Vendée) - Location of Batardières site.

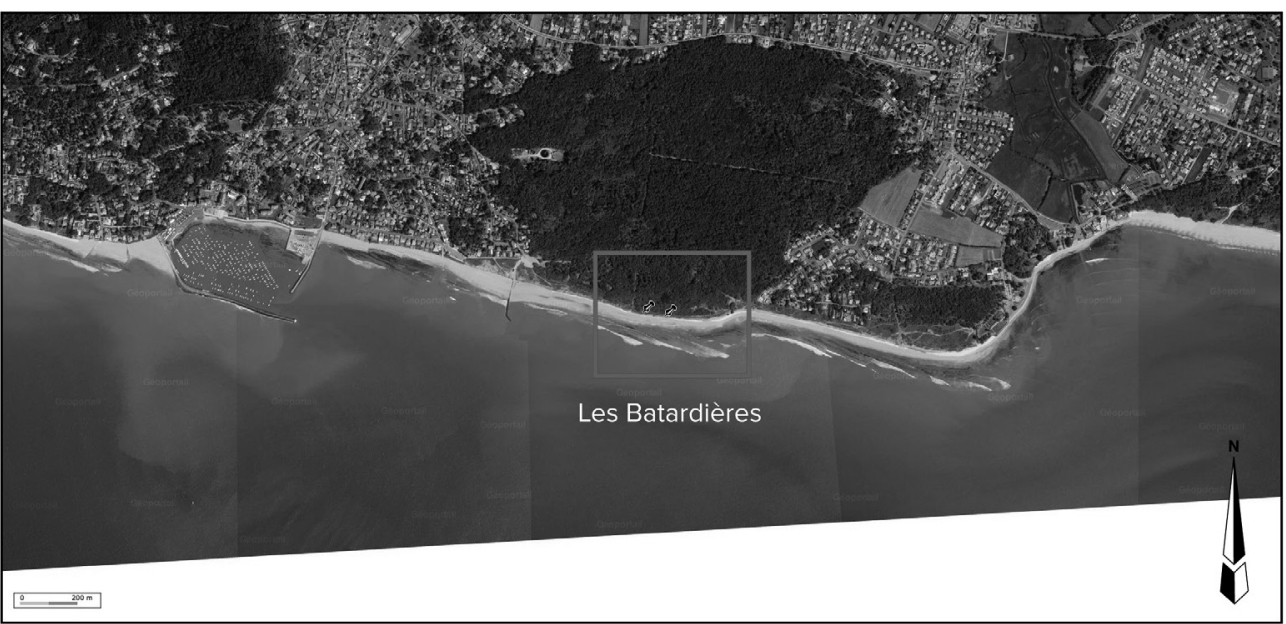

Plusieurs charbons ont été recueillis (pomoïdés, selon Nancy Marcoux ${ }^{1}$ ) et l'un d'entre eux a permis une datation radiocarbone : $2969 \pm 40 \mathrm{BP}$, soit entre 1370 et 1050 av. J.-C. (LTL 13185A), ce qui situe cette structure au tout début du Bronze final (fig. 6). Les éléments céramiques sont donc à mettre en relation avec cette période chronologique. De façon à confirmer le lien avec une exploitation du sel, des analyses de teneur en chlore sur les tessons situés au contact avec la lentille ont été effectuées. Enfin, la présence de coquillages associés implique une étude de cet ensemble.

1. Nancy Marcoux, UMR 6566 - CReAAH, Campus de Beaulieu Bât. 24, 35042 Rennes cedex - que nous remercions ici. 


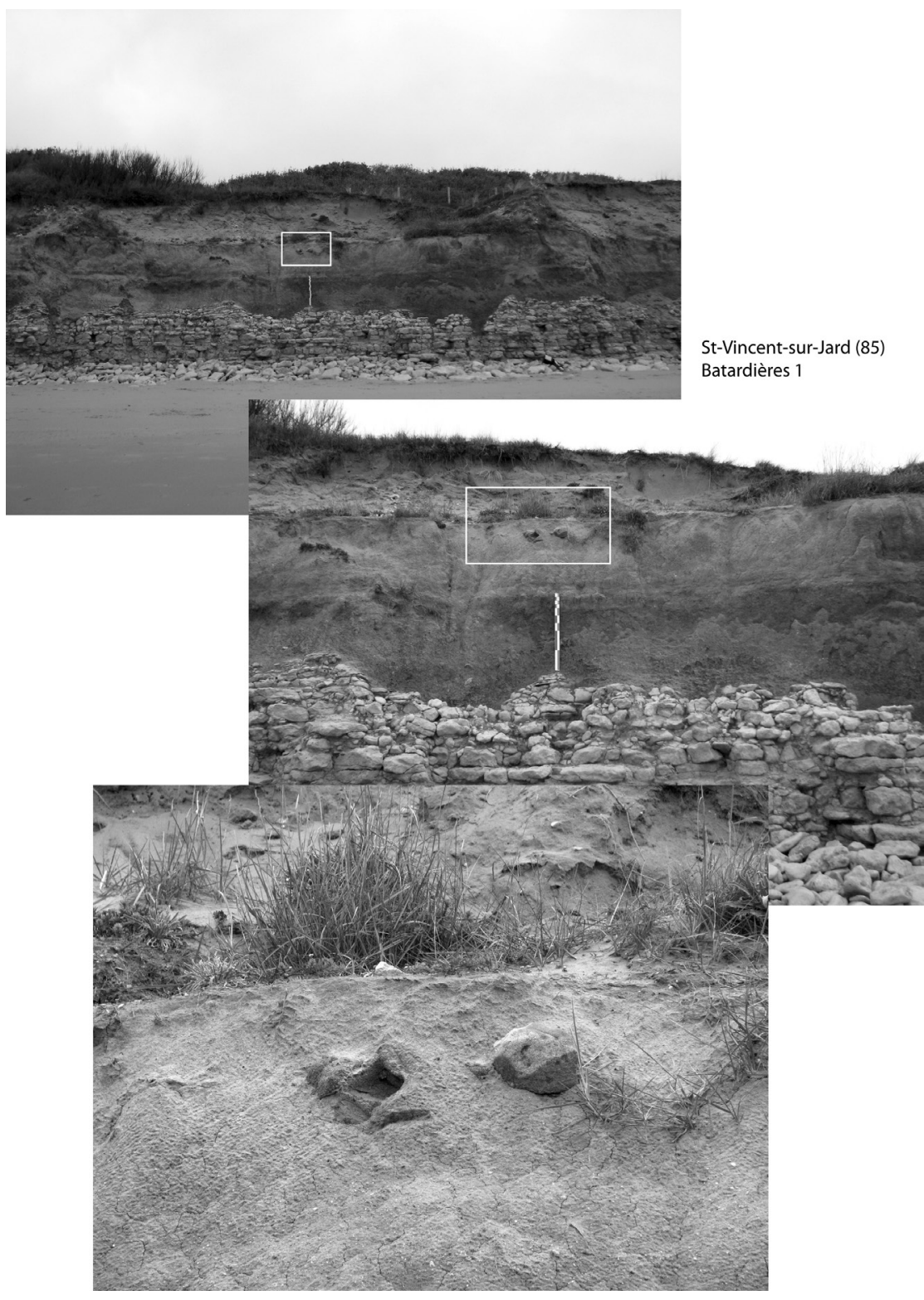

Figure 2 : Saint-Vincent-sur-Jard (Vendée) Le site de Batardières 1, situé le plus à l'ouest, comprend quelques pierres brûlées en haut de falaise. Un fragment de meule a été prélevé lors d'une intervention.

Figure 2: Saint-Vincent-sur-Jard (Vendée) - The Batardieres 1 site, located further west, includes some burnt stones on top of a cliff. A fragment of at whetstone was also removed during an archaeological excavation.
Au nord-ouest de la lentille, un grand galet de calcaire était disposé de chant, planté dans le sable brun humique, au contact avec le toit des limons périglaciaires (fig. 4f). Il est probable que ce bloc très probablement planté volontairement est en lien avec la lentille de combustion.

Les conditions de fouille étaient telles (haut de falaise, en bordure d'une terrasse de faible largeur) qu'il n'a pas été possible d'aller plus loin dans les investigations de terrain. Il est probable que cette structure de rejet fait partie d'un dispositif plus important, masqué par la dune actuelle mais qui, aussi, a peut-être disparu lors de l'effondrement de la falaise.

\section{APPROCHE DES CÉRAMIQUES PRÉSENTES}

Deux ensembles céramiques se distinguent par leur situation. Le premier concerne les quarante tessons présents sur la masse même de la lentille de combustion qui peuvent appartenir à un même récipient. Ils sont extrêmement dégradés par la combustion et par la nature même de la pâte (fig. $8, \mathrm{n}^{\text {os }} 3$ et 4 ), sans doute altérée également par sa fonction (chauffe d'une saumure). Il n'a pas été possible de connecter deux tessons en raison de l'état de dégradation et de fragilisation de leur tranche. La forme même de la céramique est donc inconnue. Tout juste pouvonsnous envisager une forme assez haute. Aucun élément de 
Figure 3 : Saint-Vincent-sur-Jard (Vendée) - Le site de Batardières 2, lui aussi disposé en haut de falaise, sur le toit des limons périglaciaires et sous la dune actuelle, laissait apparaître une lentille de combustion avec des vestiges céramiques. C'est cette structure qui a fait l'objet d'une petite intervention archéologique de terrain.

Figure 3: Saint-Vincent-sur-Jard (Vendée) - Site Batardières 2, also located on a cliff top, on a layer of periglacial silt and under the dune includes a hearth with potsherds. This feature was excavated.

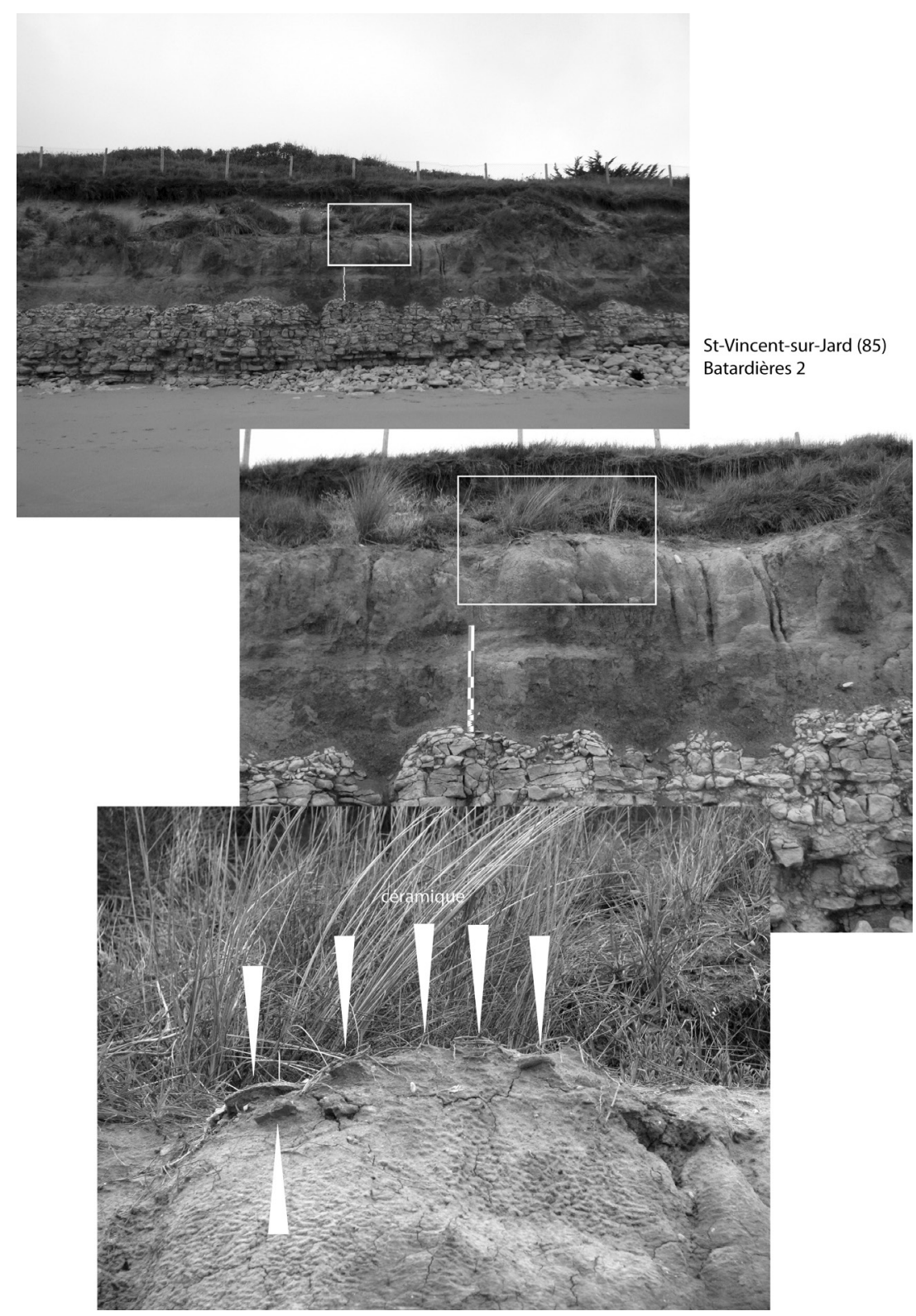

fond n'a été préservé. La couleur de la paroi externe varie du brun orangé au gris clair à foncé. La paroi interne présente une couleur brun-olive et est mieux conservée que la paroi externe, très dégradée. De nombreuses vacuoles se remarquent sur la paroi interne. L'aspect poreux de la pâte utilisée est ce que l'on retient dès la première observation. Le dégraissant utilisé pour la pâte est composé de grains de quartz abondant essentiellement roulés. Quelques fragments de coquilles sont insérés dans la pâte. On note la présence de traces organiques.

Le second ensemble regroupe des tessons de deux poteries distinctes. Il est situé à un mètre à l'ouest de la structure de combustion. Les tessons ne sont pas dans un état de dégradation aussi prononcé que pour le précédent ensemble. Toutefois, ils sont aussi très altérés. Les quelques éléments découverts lors de l'intervention ne forment pas à eux seuls des profils reconstituables. Il est probable que certains tessons ont disparu lors de l'érosion de la falaise. Les restes d'une première céramique concernent un vase à paroi légèrement convexe avec un départ d'anse en ruban (fig. $8, \mathrm{n}^{\circ} 1$ ). Il s'agit d'une forme haute qui présente malgré tout une légère inflexion. Ses parois intérieure et extérieure sont lissées par un bouchon végétal qui a laissé des traces. La couleur extérieure est brun-gris, intérieure brun-noir. Le dégraissant est formé de grains quartzeux anguleux et lisses et on note la présence de traces végétales à l'intérieur de la 

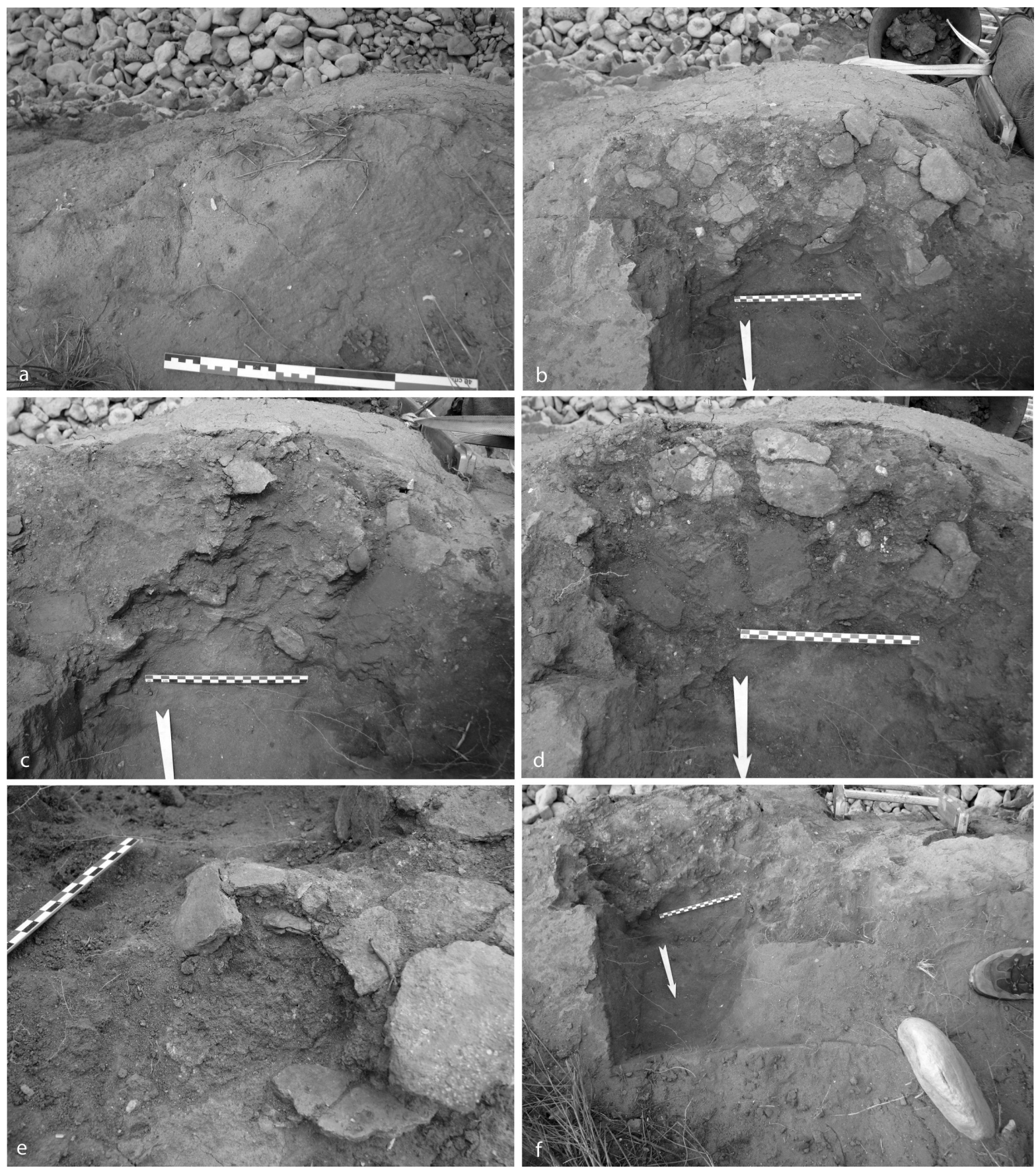

Figure 4 : Saint-Vincent-sur-Jard (Vendée) - Batardières 2 : a- Avant l'intervention, la vue zénithale de la lentille ne laisse apparaître qu'une mince couche rougie avec quelques tessons débordant d'un encroûtement brun clair; b- La première passe montre l'importance de l'ensemble céramique qui repose sur la lentille rubéfiée; c- Vue zénithale de la troisième passe. La lentille carbonisée est nettement visible sur ce cliché. Les tessons sont présents du haut en bas de la masse; d- Vue zénithale de la quatrième passe. Des restes de coquillages carbonisés apparaissent alors; e- La lentille de sédiment rubéfié apparaît alors sous les tessons. Les fragments de céramique situés à la base semblent indiquer un enveloppement de la masse carbonisée; f- Vue zénithale de l'ensemble de la fin de l'intervention. Au nord-ouest de l'emprise, le galet calcaire disposé de chant est bien visible. Il semble indiquer cette structure.

Figure 4: Saint-Vincent-sur-Jard (Vendée) - Batardières 2: a-View of the hearth before excavation, showing a thin layer reddened with some sherds on a light brown crust; $b$ - The first level shows the large amount of pottery on the reddened earth; $c$-overview of the third level. The charred fill is clearly visible in this shot. The sherds are found above and below the mass; $d$-over view of the fourth level. Charred shell remains; e- The reddened sediment appears under the sherds. The pottery at the base suggests they were stuck in a charred mass; $f$-over view of the excavation. To the Northwest, an upright limestone block is clearly visible. It marks the feature. 


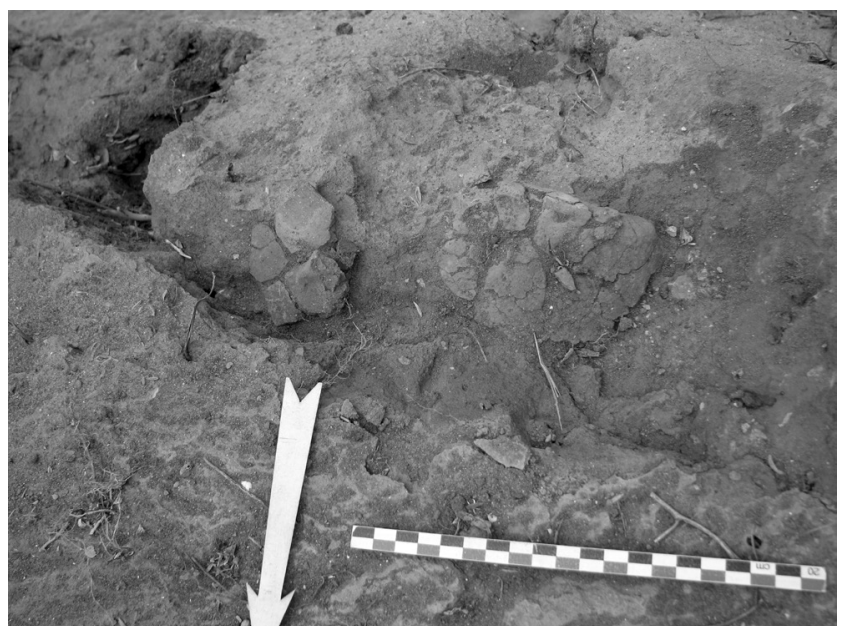

Figure 5 : Saint-Vincent-sur-Jard (Vendée) - Batardières 2 : Vue zénithale des deux amas de tessons situés à $1 \mathrm{~m}$ plus à l'ouest de la lentille de combustion.

Figure 5: Saint-Vincent-sur-Jard (Vendée) - Batardières 2: Aerial view of the two sherd clusters located $1 \mathrm{~m}$ to the west of the hearth.

pâte. L'autre élément céramique est formé d'un tesson d'un vase à paroi convexe présentant une cannelure décorative. La pâte utilisée est feuilletée, la couleur de la paroi externe est brun-gris clair, l'intérieur est brun-noir. Un reste de fond très desquamé indique une forme aplatie.

Le départ d'une anse en ruban est signifiant des céramiques du groupe des Duffaits où ce type d'anse est présente sur des vases à rupture de pente située sur le tiers supérieur du vase, sur des pots à panse sinueuse ou sur des vases de grande contenance, biconique à col élevé, appelés cruches (Bz B et C; Gomez, 2013).

La présence d'une cannelure sur le tesson (fig. $8, n^{\circ} 2$ ) n'est certes pas déterminante mais se retrouve dans le groupe des Duffaits.

Les deux ensembles céramiques rapidement décrits sont donc conformes à la datation au radiocarbone et positionnent la structure de combustion au tout début du Bronze final (BF1a ou BzD1) à la suite de l'ultime phase de la culture des Duffaits (Gomez, 2013).

\section{UNE EXPLOITATION DU SEL DÉMONTRÉE : ANALYSES CHIMIQUES DE LA CÉRAMIQUE}

Pour répondre à la question d'une exploitation du sel fortement soupçonnée sur le site des Batardières 2, quatre échantillons ont été testés et analysés chimiquement. Ils ont été prélevés en juin 2013 par Vincent Ard parmi le matériel fouillé suivant le protocole mis au point lors des études sur la production de sel du Néolithique récent autour du Marais poitevin (Weller, 2004; Ard et Weller, 2012).

\section{Méthode d'analyse}

Sans revenir en détail sur le protocole et la méthode d'analyse (voir Ard et Weller, 2012), il s'agit en résumé de mesurer et comparer dans différents échantillons les teneurs en chlore obtenus en XRF (spectrométrie de fluorescence X en laboratoire, limite de détection autour de $50 \mathrm{ppm})$. Ces échantil-

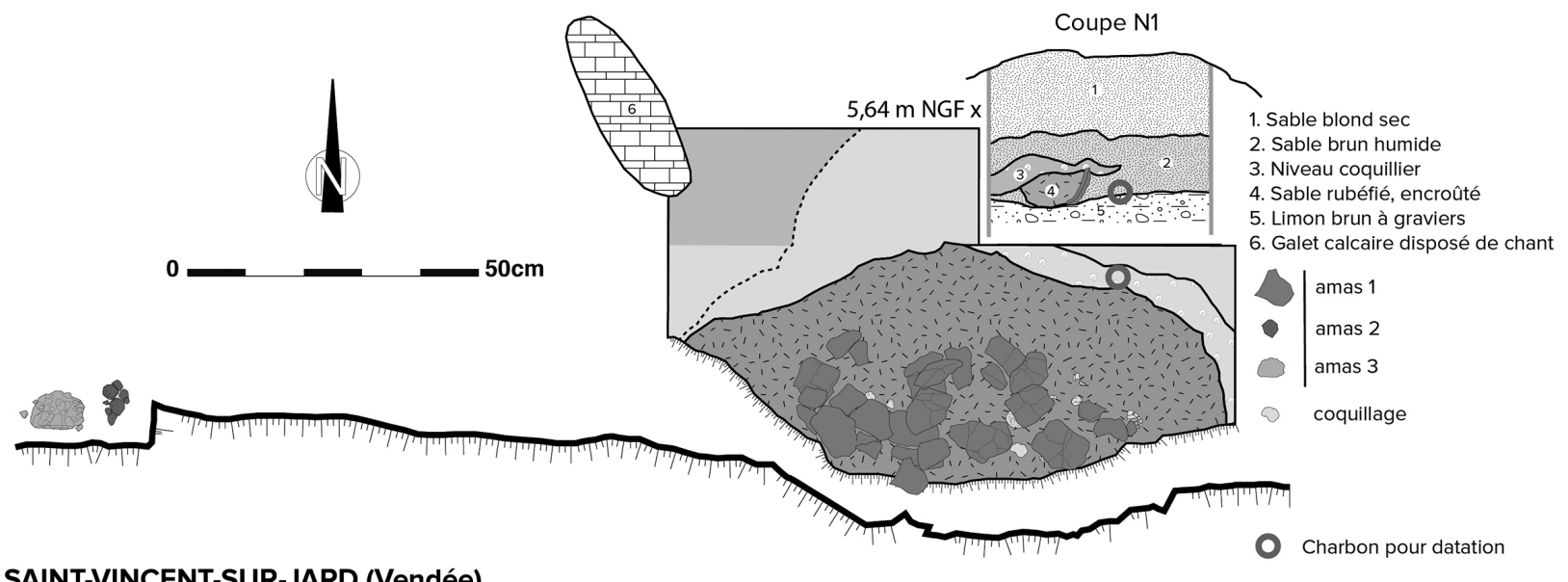

\section{Les Batardières 2}

$X$ du centroïde : $\quad 300.297$

Y du centroïde : $\quad 2163.724$

Z du centroïde : $\quad 5,64$ m NGF

$N: 46^{\circ} 24^{\prime} 23.6^{\prime \prime} \quad W: 01^{\circ} 33^{\prime} 44.8^{\prime \prime}$
Figure 6 : Saint-Vincent-sur-Jard (Vendée) - Batardières 2 : Plan général de l'intervention et coupe au nord de la structure. Figure 6: Saint-Vincent-sur-Jard (Vendée) - Batardières 2: General plan of the excavation and cut to the north of the feature. 


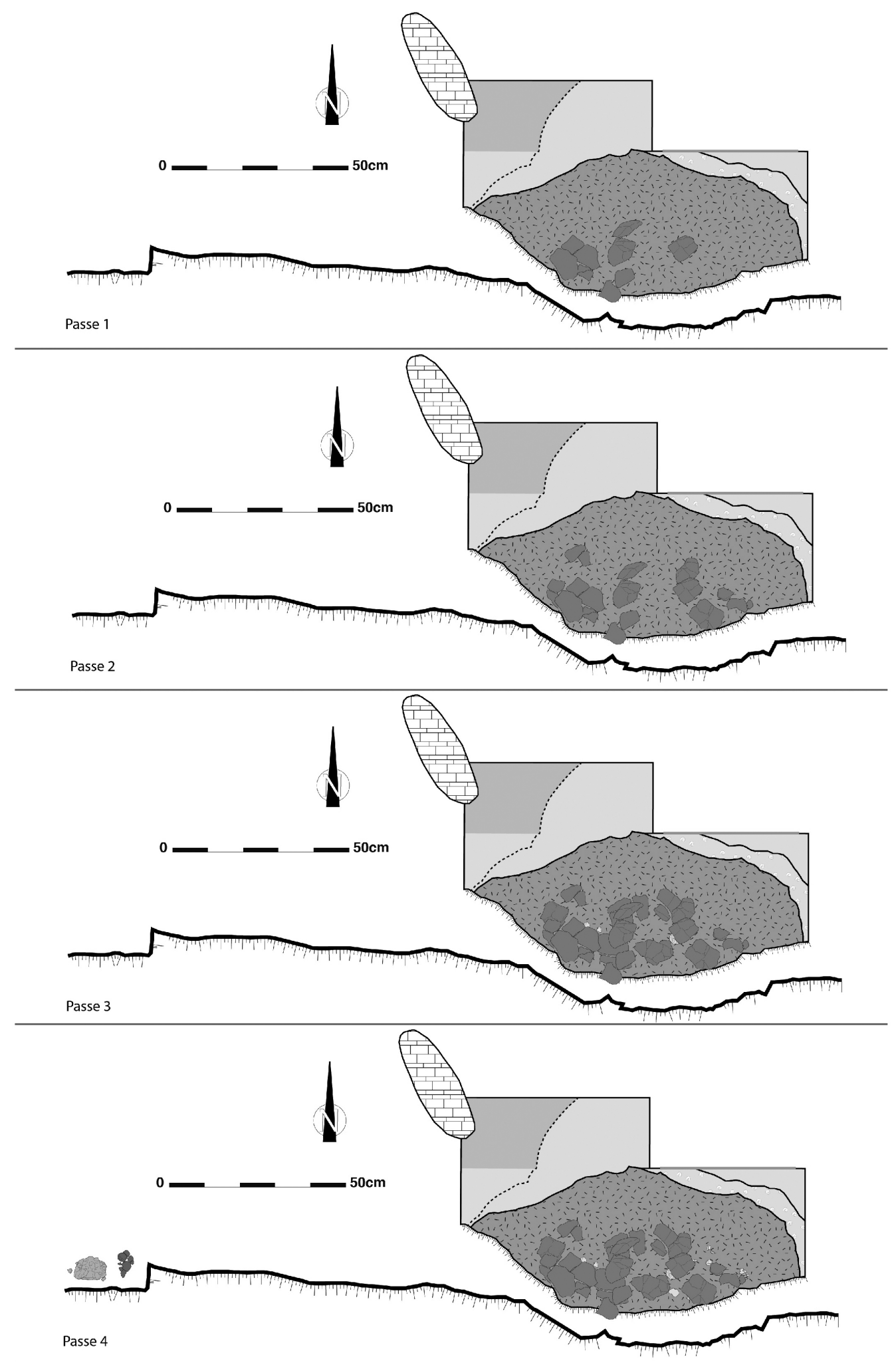

Figure 7 : Saint-Vincent-sur-Jard (Vendée) - Batardières 2 : Plan cumulé des différentes passes de fouille montrant la dispersion des vestiges céramiques (légende de la Figure 7).

Figure 7: Saint-Vincent-sur-Jard (Vendée) - Batardières 2: Map bringing together the different excavation levels showing the distribution of the pottery (legend of Figure 7). 

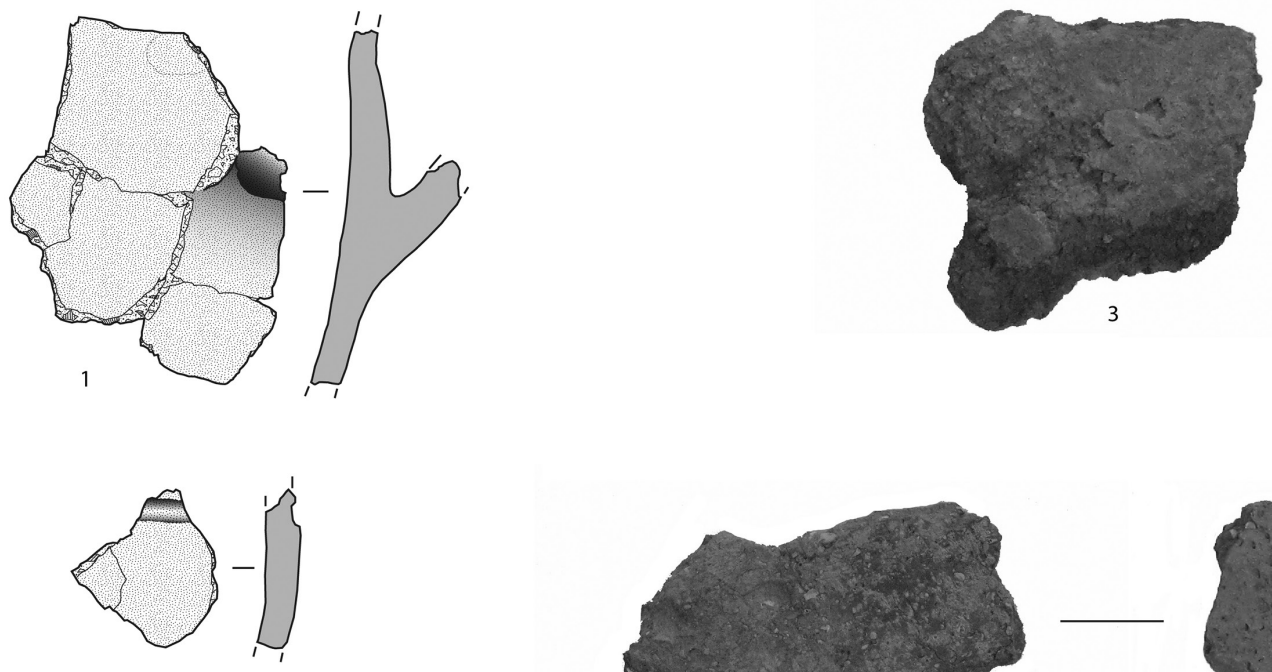

2
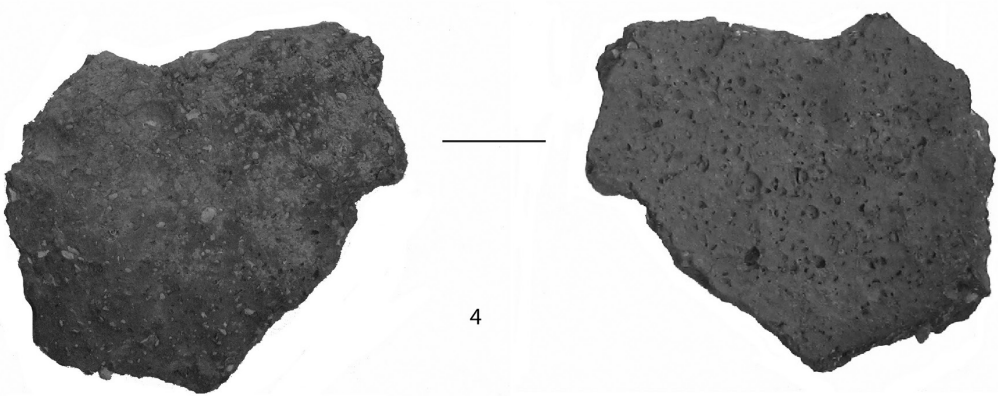

Figure 8 : Saint-Vincent-sur-Jard (Vendée) - Batardières 2 : Mobilier céramiques. 1- Fragment d'un vase à paroi ovoïde avec départ d'anse en ruban; 2- Tesson d'un autre vase avec présence d'une cannelure décorative; 3 et 4-État de surface de tessons de l'amas rubéfié. La recuisson des tessons a provoqué des éclats thermiques. La face interne montre de nombreuses vacuoles.

Figure 8: Saint-Vincent-sur-Jard (Vendée) - Batardières 2: Pottery 1-Fragment of an ovoid vase with ribbon handle; 2- Sherd from another vessel with decorative groove; 3 and 4 - The surface of the sherds found in the reddened clusters. The re firing of the sherds has caused thermal chips. The internal surface shows many vacuoles.

lons sont préalablement réduits en poudre (minimum $1 \mathrm{~g}$ ) puis compressés sous forme de pastilles permettant d'obtenir une surface bien plane face aux rayonnements dans le spectromètre; les mesures sont centrées sur l'élément chimique et le temps de comptage prolongé jusqu'à 10 minutes par échantillon. En sélectionnant, à côté des possibles vases à sel, du sédiment enrobant et des éléments de production céramique domestique présentant le même type de pâte et provenant, si possible, des mêmes niveaux archéologiques, on peut comparer les teneurs en chlore où la céramique domestique et le sédiment enrobant servent de points de référence.

Dans le cas du site des Batardières 2, la prise en compte précoce de la question de l'exploitation du sel a permis d'optimiser l'échantillonnage avec à la fois, comme élément de référence, un prélèvement de sédiment du foyer contenant quelques nodules d'argile et un tesson de panse provenant d'un vase domestique à anse décoré de cannelures et présentant une pâte fine à inclusions sableuses (secteur W à $1,10 \mathrm{~m}$ du foyer principal). Pour les possibles vases à sel, deux échantillons représentatifs ont été analysés au sein du foyer, respectivement dans les passes 2 et 3 . Le premier est un tesson de haut de panse présentant une pâte poreuse, de fines inclusions de quartz et de calcaire et des surfaces altérées; le second, un tesson de panse avec une pâte très poreuse, des inclusions coquillées et organiques et des surfaces altérées.

Afin de tester et affiner notre méthode, nous avons couplé les mesures des teneurs en chlore avec celles du brome (limite de détection autour de $10 \mathrm{ppm}$ ), un élément chimique mineur mais discriminant car il est contenu naturellement dans l'eau de mer et n'existe principalement que sous forme liquide (bromure).

Les analyses ont été réalisées en septembre 2013 en partenariat avec le Service Central d'Analyses (UMR 5280 du CNRS, Villeurbanne).

\section{Résultats}

Les résultats obtenus (fig. 9) montrent tout d'abord que les teneurs en chlore ou en brome varient plus que de 1 à 5 selon les échantillons. Les échantillons de référence (sédiment et céramique domestique) ne renferment que de très faibles quantités de chlore et très peu de brome (limite de 
détection autour de $10 \mathrm{ppm}$ ). En revanche, les deux fragments de probables vases à sel montrent des concentrations en chlore, comme en brome, deux à cinq fois plus importantes. Ces plus fortes concentrations ne peuvent s'expliquer par une pollution post-dépositionnelle et l'adéquation des mesures obtenues pour le chlore et le brome renforce le fait que ces deux fragments ont bien été en contact avec une solution d'eau de mer concentrée ou non. Ajoutons que ces tessons à l'aspect vacuolaire, aux surfaces altérées non lissées, parfois dégraissés à l'aide de coquilles ou de matière organique, évoquent fortement ceux décrits pour la production de sel du Néolithique récent, en particulier ceux du sud du Marais poitevin comme à La Sauzaie ou La Mastine (Ard et Weller, 2012).

Bien que réduite, la série d'analyses a permis de confirmer l'efficience de la méthode de détection du sel à travers la mesure croisée des teneurs en chlore et en brome dans d'anciennes céramiques, y compris dans un contexte de falaise littorale soumise aux embruns et aux pollutions marines.

\section{LE PETIT LOT DE COQUILLES DE LA STRUCTURE DE COMBUSTION}

Les prélèvements effectués sont localisés dans le foyer luimême et sa périphérie (tableau 1).

Ce foyer est attribué de la fin du Bronze moyen au début du Bronze final. Malgré les faibles quantités d'invertébrés marins découverts et étudiés, ce témoignage de l'exploitation des ressources marines est précieux. En effet, les informations concernant l'exploitation des coquillages marins pour les périodes concernées sont extrêmement rares sur le littoral atlantique français et inexistantes sur la côte vendéenne (Mougne, à paraître).

\begin{tabular}{|l|l|}
\hline US & Passe \\
\hline Périphérie du foyer & 1 \\
\hline Prélèvement de coquilles & Base et périphérie est du foyer \\
\hline Poche 1 & 3 \\
\hline Poche 2 & 3 \\
\hline \multirow{2}{*}{ Foyer } & 2 \\
\cline { 2 - 2 } & 3 \\
\cline { 2 - 2 } & 4 \\
\hline Niveau de coquillages & Partie est du foyer \\
\hline
\end{tabular}

Tableau 1 : Liste des échantillons ayant livré des invertébrés marins : les Batardières 2.

Table 1: list of samples containing marine invertebrates: Les Batardières 2.

\section{Méthodologie}

Les échantillons étudiés correspondent à des prélèvements coquilliers faits avec leur sédiment. Ceux-ci ont été tamisés et étudiés. L'intégralité des lots a été prise en compte.

Au sein de chaque échantillon, les fragments coquilliers d'une même espèce ont été regroupés entre eux. Ils ont été pesés et décomptés en NR ou nombre de restes lorsque leur dimension était supérieure à $2 \mathrm{~mm}$. Le NMI ou nombre minimum d'individu a également été calculé. Pour les gastéropodes coniques, comme la patelle, a été compté comme un individu, tous les tests présentant un apex associé à une partie de l'empreinte musculaire. Celle-ci s'observe à l'intérieur du test. Cette précaution se justifie par le dédoublement possible de l'apex lors de la dégradation dans le sol du test coquillier. Pour les bivalves, un NMI par combinaison est utilisé. Il est obtenu après latéralisation des valves. Celle-ci est appliquée en fonction, entre autres, de la localisation des dents de la charnière et celle du sinus palléal lorsqu'il est présent. Ainsi, un échantillon se composant de vingtquatre valves droites et de dix-huit valves gauches le NMI sera estimé à 24 . Les fragments coquilliers qui présentent des indices macroscopiques de calcination ont été notés sous le terme nombre de restes brûlés ou NB.

Lidentification des mollusques est fondée sur la forme de la coquille, sa charnière pour les bivalves, son ornementation, ainsi que les empreintes laissées sur la coquille par la chair de l'animal, notamment les empreintes musculaire et ligamentaire. Les appellations scientifiques utilisées ou noms latins changent régulièrement. Celles utilisées dans le cadre de ce rapport sont issues du CLEMAM (Check List of European Marine Mollusca, 2013). L'identification des coquillages s'est faite à partir d'ouvrages de référence (Poppe et Goto, 1991, 1993). Tous les tests complets ont été mesurés ainsi que, sur les exemplaires incomplets, les zones des coquilles permettant la reconstitution de leurs tailles originelles. Les fragments calcinés ont aussi été décomptés. Ils se matérialisent par une coloration grise associée à des microfissures de la coquille.

\section{Résultats}

Au total, $529 \mathrm{~g}$ de coquilles ont été étudiés pour 108 individus et 622 fragments coquilliers (tableau 2).

Cinq espèces de mollusques ont été déterminées : trois gastéropodes, représentés par des patelles ou berniques Patella vulgata, $P$. depressa et $P$. ulyssiponensis et deux bivalves, la scrobiculaire Scrobicularia plana et la coque Cerastoderma sp. Deux crustacés ont aussi été identifiés : les crustacés cirripèdes représentés par les balanes Balanus sp. et les Chtamalus sp. et les crustacés décapodes ou crabes pour lesquels le tour- 

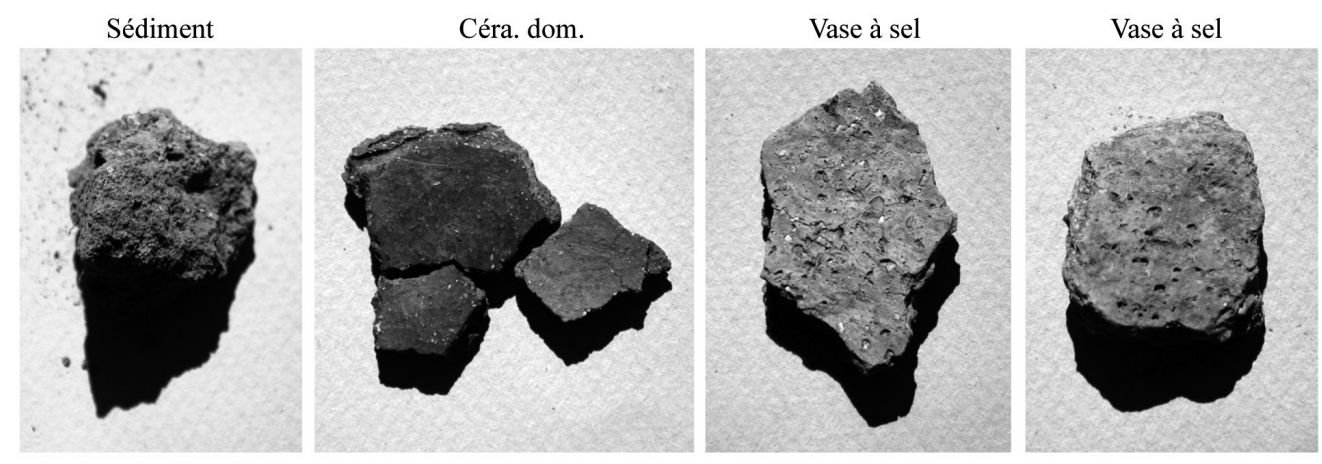

Vase à sel probable (tesson de haut de panse)

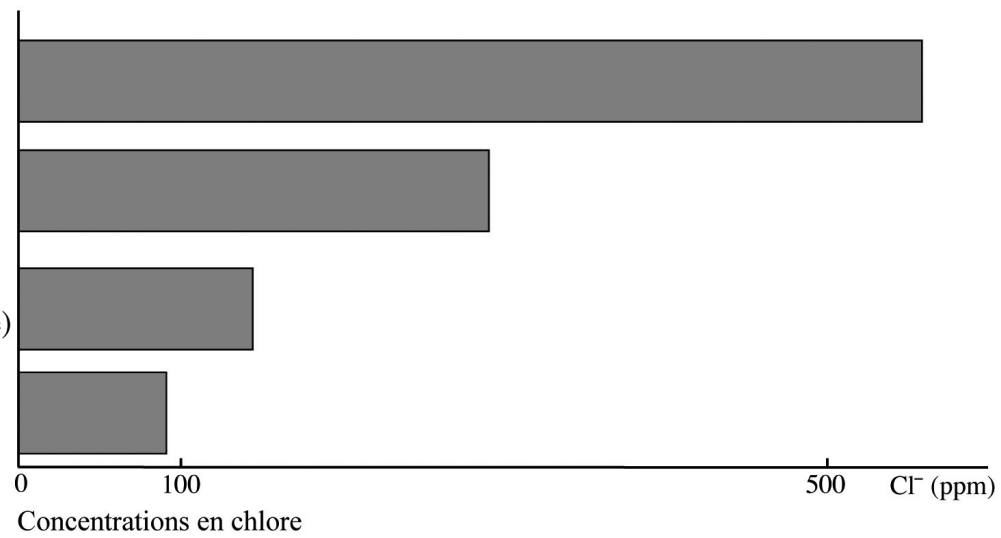

Vase à sel probable (tesson de panse très poreux)

Céramique domestique (tesson de panse de vase à anse)

Sédiment archéologique (foyer)

Concentrations en chlore

Vase à sel probable (tesson de haut de panse)

Vase à sel probable (tesson de panse très poreux)

Céramique domestique (tesson de panse de vase à anse)

Sédiment archéologique (foyer)

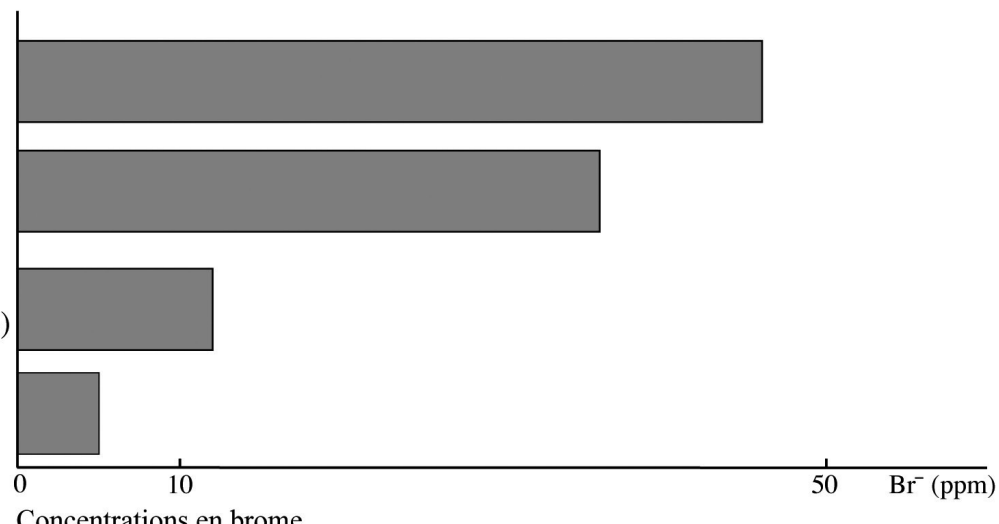

Figure 9 : Saint-Vincent-sur-Jard (Vendée) - Batardières 2 : Analyses chimiques en XRF des teneurs en chlore et en brome sur 4 échantillons (1 sédiment, 3 tessons céramique). SCA-CNRS, UMR 5280, Villeurbanne, sept. 2013, clichés et DAO O. Weller.

Figure 9: Saint-Vincent-sur-Jard (Vendée) - Batardières 2: XRF chemical analysis of chlorine and bromine concentrations of 4 samples (1 sediment, ceramic sherds 3).

teau ou dormeur Cancer pagurus a été observé (tableau 2, fig. 10). Tous ces coquillages ne sont pas représentés équitablement. En effet, les patelles dominent largement le corpus avec un pourcentage de $98 \%$ si leur masse est prise en compte et $91 \%$ pour le NMI. Elle est suivie par la scrobiculaire dont seulement six individus ont été identifiés.

L'état de fragmentation des coquilles est tel que seulement dix-sept longueurs de patelles ont pu être mesurées pour quatre-vingt-dix-huit individus identifiés. Cette fragilisation des tests a largement pu être accentuée par le contact d'artefacts avec le feu $(\mathrm{NB}=622)$. Ainsi, la longueur moyenne obtenue pour ces dix-sept patelles est de $33 \mathrm{~mm}$ pour une valeur minimale de $29 \mathrm{~mm}$ et une maximale de $40 \mathrm{~mm}$. Ces mesures correspondent à la sélection d'individus de grandes dimensions sur l'estran. Ce résultat est renforcé par le fait que ces coquilles proviennent de prélèvements sédi- 


\begin{tabular}{|c|c|c|c|c|c|c|}
\hline Liste des espèces & Masse $(\mathrm{g})$ & NR & NMI & Valve droite & Valve gauche & NB \\
\hline \multicolumn{7}{|l|}{ Crustacés } \\
\hline \multicolumn{7}{|l|}{$\begin{array}{l}\text { Crustacés cirri- } \\
\text { pères }\end{array}$} \\
\hline Balanus sp. & 0,01 & 1 & 1 & - & - & \\
\hline Chtamalus sp. & 0,01 & 1 & 1 & - & - & \\
\hline \multicolumn{7}{|l|}{$\begin{array}{l}\text { Crustacés déca- } \\
\text { podes }\end{array}$} \\
\hline Cancer pagurus & 0,6 & 4 & 1 & - & - & \\
\hline \multicolumn{7}{|l|}{ Mollusques } \\
\hline \multicolumn{7}{|l|}{ Bivalves } \\
\hline Scrobicularia plana & 8,55 & 240 & 6 & 6 & 4 & 27 \\
\hline Cerastoderma sp. & 0,06 & 2 & 1 & 0 & 0 & \\
\hline Gastéropodes & & & & - & - & \\
\hline Patella sp. & 519,99 & 4038 & 98 & - & - & 594 \\
\hline Indéterminé & 0,05 & 3 & 0 & - & - & 1 \\
\hline Total & 529,27 & 4289 & 108 & 6 & 4 & 622 \\
\hline
\end{tabular}

Tableau 2 : Bilan quantitatif des invertébrés marins déterminés dans la fosse « Les Batardières 2 ». Table 2: Quantitative results for marine invertebrates identified in "Les Batardières 2" trench.

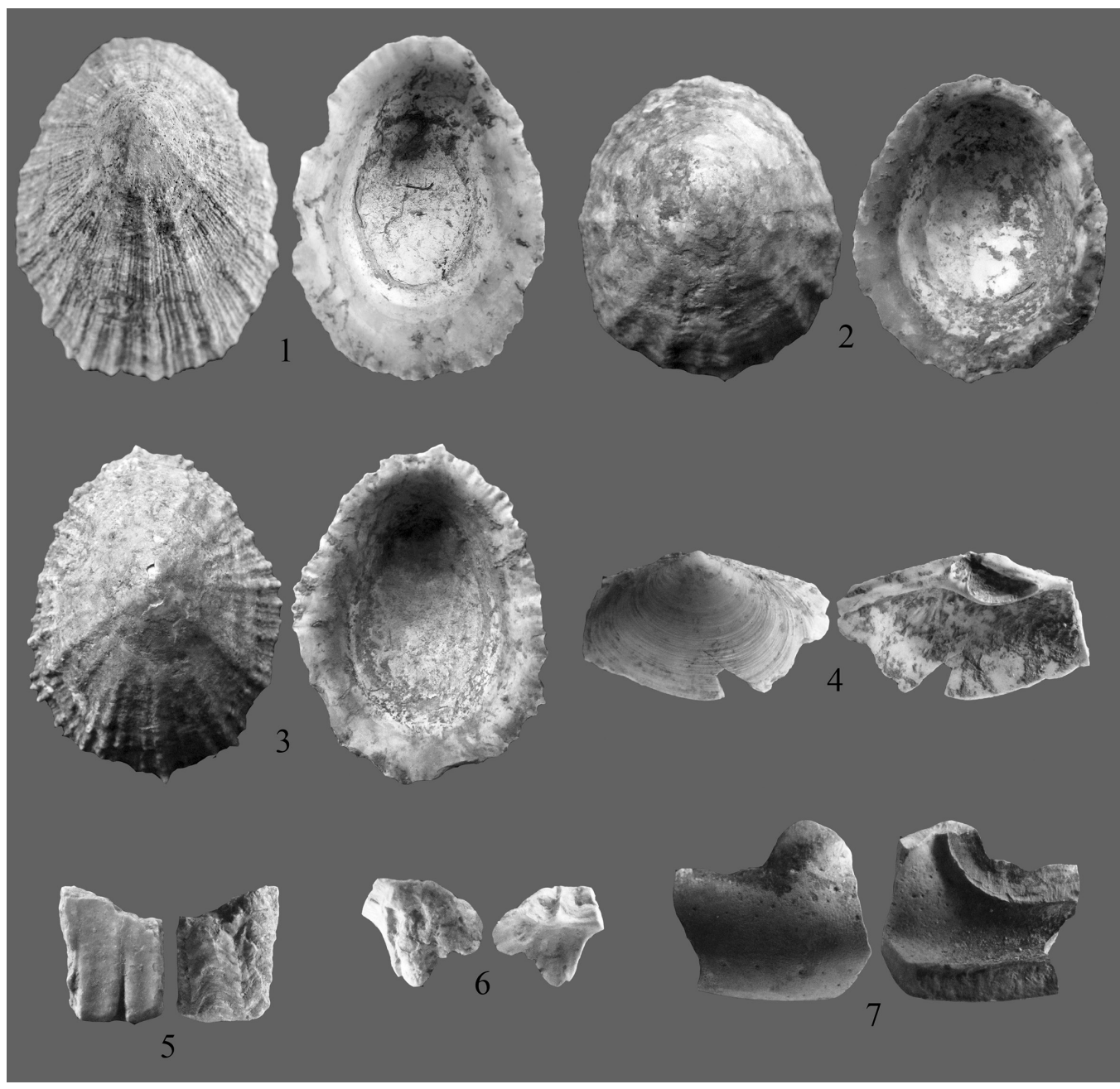

Figure 10 : Saint-Vincentsur-Jard (Vendée) - Batardières 2 : Les invertébrés marins. 1 Patella vulgata $(36 \mathrm{~mm}), 2$ Patella depressa $(33 \mathrm{~mm})$,

3 Patella ulyssiponensis (40 mm), 4 Scrobicularia plana $(15 \mathrm{~mm})$, 5 Cerastoderma sp. (7 mm), Chtamalus sp. (3 $\mathrm{mm})$, Cancer pagurus $(10 \mathrm{~mm})$ (Clichés et DAO C. Dupont). Figure 10: Saint-Vincentsur-Jard (Vendée) Batardières 2: Marine invertebrates. 1 Patella vulgata $(36 \mathrm{~mm}), 2$ Patella depressa $(33 \mathrm{~mm})$, 3 Patella ulyssiponensis $(40 \mathrm{~mm}), 4$ Scrobicularia plana $(15 \mathrm{~mm}), 5$ Cerastoderma sp. $(7 \mathrm{~mm})$, Chtamalus sp. $(3 \mathrm{~mm})$, Cancer pagurus $(10 \mathrm{~mm})$ (Photographs and DAO C. Dupont). 
mentaires et donc que cette sélection n'a pas été accentuée par la méthode de fouille. La scrobiculaire est un bivalve extrêmement fragile. Pour avoir accès à sa longueur originelle, celle de son empreinte ligamentaire peut être mesurée (Dupont, 2006). C'est ce qui a pu être réalisé sur deux valves gauches et cinq valves droites de scrobiculaires sur "Les Batardières 2 ». Ces sept valves ont une moyenne de $39 \mathrm{~mm}$ pour un minimum de $33 \mathrm{~mm}$ et un maximum de $46 \mathrm{~mm}$. Comme pour les patelles, les scrobiculaires représentées sur "Les Batardières 2 " sont des individus de grande dimension. Ces coquillages ont potentiellement pu être collectés pour leurs grandes dimensions dans le but d'être mangés. Le fragment de pince de tourteau ne correspond pas à un crabe juvénile. Ce crustacé aurait très bien pu avoir été consommé.

Un premier constat peut-être fait concernant les environnements dont proviennent ces coquillages : les espèces les mieux représentées ne vivent pas dans ou sur les mêmes substrats (fig. 11). En effet, les patelles vivent accrochées sur les rochers tandis que les scrobiculaires vivent enfouies dans les vasières. Toutes deux ont été principalement identifiées dans la passe 4 et la périphérie est du foyer. Ce contraste entre leurs biotopes est assez étonnant au vu du faible nombre de restes étudiés, mais il est possible que ces coquillages ne soient qu'un petit échantillon d'un assemblage qui était beaucoup plus vaste.

Parmi les patelles, seules vingt ont pu être déterminées : une Patella vulgata, trois $P$. depressa et seize $P$. ulyssiponensis. Ces proportions correspondent à l'exploitation d'un moyen estran (fig. 11).

Les fragments coquilliers et de crustacés présentant des indices de calcination macroscopiques (coloration grise) ont été décomptés en fonction de leur position stratigraphique (tableau 3). Le résultat montre une concentration de ces fragments brûlés dans les passes 1 et 2 du foyer qui diminue

\begin{tabular}{|c|c|c|c|c|c|}
\hline \multirow{2}{*}{ Les espèces } & \multirow[t]{2}{*}{ Substrat } & \multicolumn{3}{|c|}{ Intertidale } & \multirow{2}{*}{ Subtidale } \\
\hline & & Haut estran & Moyen estran & Bas estran & \\
\hline Patella intermedia & \multirow{3}{*}{ roche } & & & & \\
\hline Patella ulyssiponensis & & & & & \\
\hline \multicolumn{5}{|l|}{ Patella vulgata } & \\
\hline Cerastoderma sp. & Sable envasé à vase & & & & \\
\hline Scrobicularia plana & vase & & & & \\
\hline \multicolumn{2}{|c|}{ H.M.V.E. } & & & & \\
\hline \multicolumn{6}{|c|}{$0 \mathrm{~m}$ N.G.F. } \\
\hline B.M.V & & & & & \\
\hline
\end{tabular}

Figure 11 : Localisation potentielle des espèces déterminées sur "Les Batardières 2 " (H.M.V.E. : Haute Mer de Vive Eau; N.G.F. : Nivellement géographique de la France; B.M.V.E. : Basse Mer de Vive Eau, case grisée : présence potentielle). D’après Dupont et Gruet 2001 ; Dupont 2006.

Figure 11: Potential location of specific species on "Batardières 2" (HMVE: High Sea Water Vive; NGF: Geographical Leveling of France LLW: Low Sea Water Vive, shaded box: potential presence).

Tableau 3 : Pourcentage de calcination identifiés à vue sur les invertébrés marins déterminés dans la fosse «Les Batardières 2 ». Table 3: Statistics for calcinated (from visual observations) marine invertebrates identified in "Les Batardieres 2" trench.

\begin{tabular}{|l|c|c|c|}
\hline Référence de l'échantillon & NR & NB & $\%$ NB \\
\hline BA2012-Périphérie Foyer Passe 1 & 280 & 86 & 31 \\
\hline BA2012-Foyer Passe 2 & 247 & 78 & 32 \\
\hline BA2012-Foyer Passe 3 & 1670 & 270 & 16 \\
\hline BA2012-Foyer Passe 4 & 854 & 72 & 8 \\
\hline BA2012-Poche2 Passe 3 & 372 & 63 & 17 \\
\hline BA2012-Poche1 Passe 3 & 247 & 40 & 16 \\
\hline BA2012-Partie Est Foyer & 259 & 7 & 3 \\
\hline BA2012-Base et Périphérie Est Foyer & 360 & 8 & 2 \\
\hline
\end{tabular}


progressivement des passes 3 à 4 . Cette « stratigraphie » des restes d'invertébrés marins correspond sans doute à leur calcination in situ après leur consommation. Cette observation est liée au fait que les coquilles n'étaient pas positionnées au cœur du foyer mais dans sa périphérie. En effet, la coupe N1 montre que les coquilles les plus carbonisées (3. niveau coquillier, fig. 6) sont sur la zone rubéfiée.

\section{QUELLE FONCTION DONNER À LA STRUCTURE DE COMBUSTION DES BATARDIÈRES 2?}

En Poitou, la connaissance des implantations humaines au Bronze moyen/final est très partielle (Joussaume et Pautreau, 1990). Sont connus essentiellement des restes d'habitats ou des traces d'activités artisanales dans des zones de vallées, sous forme de série de fosses autour d'un foyer à Viaube de Jaunay-Clan (Vienne), interprétées comme des structures de stockage abandonnées ou transformées en dépotoir. À Civaux (Vienne), une autre fosse remplie de cendres contenait des os d'animaux et des poteries. Ensuite, ce sont des pointements qui ont permis la découverte de restes de céramiques qui témoignent de la présence d'occupations du Bronze moyen. Les sites de hauteur révèlent rarement dans leur stratigraphie un niveau Bronze moyen.

Bien qu'aucune forme n'ait pu être identifiée, la présence de vases à sel ne fait aucun doute sur le site des Batardières 2 comme le montrent les observations technologiques et les analyses chimiques réalisées sur cette production céramique particulière. La structure de combustion révélée par la tempête Xynthia pourrait bien correspondre à un ancien fourneau à sel et les vases identifiées à d'anciens moules, mais les témoins disponibles restent encore limités. Il n'en reste pas moins que le site des Batardières 2 nous permet d'envisager plus précisément une production de sel marin dès le début du Bronze final dans un contexte documentaire où l'exploitation du sel durant l'âge du Bronze moyen-final reste encore peu et mal documentée sur le littoral atlantique avec seulement quelques découvertes éparses (Curnic à Guissény, Boucaud à Préfailles, Pontreau 2 à Beauvoir-sur-Mer). En revanche, elle est mieux connue autour des sources salées de l'intérieur des terres (Fontaines Salées à Saint-Père-sousVézelay en Bourgogne, sources salées du Jura, vallée de la Seille en Lorraine, Salies-de-Béarn et Salies-du-Salat dans les Pyrénées). Comme il a été possible de le démontrer dans le Jura, l'exploitation du sel semble bien connaître un véritable essor dès le xve siècle avant notre ère en lien avec une reprise démographique (Pétrequin et Weller, 2007), essor que l'on peut illustrer également, plus loin, avec les tout débuts de l'extraction souterraine de sel gemme à Hallstatt au Xve siècle, sel destiné, entre autres, à la production de porc salé (Reschreiter et Kowarik, 2009). Qu’en était-il alors sur le littoral atlantique?

Quelques coquillages ont été découverts dans le foyer « Les Batardières 2 " daté de la fin du Bronze moyen au début du Bronze final. Ce site archéologique, malgré son état de dégradation important et le peu de restes qui subsistent, est intéressant à prendre en considération, car il est un des rares témoins de l'exploitation des invertébrés marins à l'âge du Bronze sur le littoral atlantique français. Que signifie ce petit lot de coquillages? Il peut très bien correspondre à la consommation de patelles et de scrobiculaires à la fin du fonctionnement du foyer, soit crues, soit cuites dans le foyer lorsque les braises sont encore chaudes. Ce comportement est connu pour les fours à goémons pour lesquels, à la fin de l'activité, la chaleur du four est utilisée pour cuire les coquillages, qui seront ensuite rejetés dans le foyer (Arzel, 1987, p. 211). La patelle et la plupart des coquillages se prêtent bien à ce type de cuisson car le corps de l'animal se détache rapidement de la coquille dès exposition à une source de chaleur. La dimension des coquillages observés et leur détermination montrent qu'ils ont été sélectionnés pour être consommés dans une zone rocheuse et une vasière. Les taux de calcination observés diminuent progressivement du haut vers le bas de l'accumulation coquillière. Ce gradient de stigmates de carbonisation tend à montrer que ces restes coquilliers ont donc été soumis à une source de chaleur après leur consommation. Leur rejet en périphérie de la source de chaleur la plus intense explique cette "stratigraphie » des stigmates de carbonisation.

La structure de combustion des Batardières s'inscrit dans un contexte peu disert. Récemment, les travaux d'archéologie préventive effectués sur le site du Pontreau 2 à Beauvoirsur-Mer, au nord-ouest du département de la Vendée, ont livré, entre autres, des structures fossoyées contemporaines du foyer des Batardières (Viau, 2005). Parmi cet ensemble domestique et artisanal, trois fosses ont été interprétées comme des bassins permettant le stockage de la saumure afin d'exploiter le sel marin (Daire, 2003). L'argument principal est donné par la nature du fond des cuves, composé d'argile ou de limon plastiques et par la forme de nombreuses céramiques de type godets. Malgré l'absence de rubéfaction, l'hypothèse que ces fosses aient pu servir à chauffer la saumure (par une température très basse) est avancée. Toutefois, l'absence de foyers sensu stricto en raison de la disparition des sols d'occupation ne permet pas d'avoir une représentation claire du cadre artisanal de production de sel sur ce site. Comme aux Batardières, ce site est un des rares témoins de la production du sel sur le littoral ancien au tout début du Bronze final.

Plus au nord du Marais breton, à la Roussellerie (SaintMichel-Chef-Chef, Saint-Brévin-les-Pins), les prospections 
de Michel Tessier ont permis de distinguer plusieurs locus dans des unités sédimentaires sous-dunaires dans lesquelles certaines structures ont été liées à l'activité d'exploitation du sel. Plusieurs céramiques témoignent également de cette activité dans une période chronologique similaire à celle du foyer des Batardières et du site du Pontreau (Tessier et Bernard, 1995) ${ }^{2}$.

Les Batardières 2 s'ajoutent aux quelques sites connus et apportent un élément supplémentaire aux contextes géographiques d'occupation de la transition Bronze moyen Bronze final. Il s'agit d'un site littoral qui témoigne d'une activité artisanale centrée sur l'exploitation du sel ${ }^{3}$ mais aussi d'une autre activité à des fins culinaires. Le foyer avait donc des fonctions multiples et s'insère dans un réseau de réalisations et sans doute d'échanges lié à l'exploitation du sel par chauffage de la saumure, sans doute continue depuis le Néolithique moyen.

\section{Bibliographie}

Ard V. et Weller O., 20I 2 - "La céramique, 2. Les vases de "type Champ-Durand" : témoins d'une exploitation du sel au Néolithique récent dans le Marais poitevin ", in Joussaume R. (dir.), L'enceinte néolithique de Champ-Durand à Nieulsur-l'Autize (Vendée), Éditions des Publication Chauvinoises (APC), mémoire XLIV, p. 319-343.

Arzel P., I987 - Les goémoniers, Douarnenez, Le Chasse-Marée, $305 \mathrm{p}$.

Clemam, 201 3 - Check List of European Marine Mollusca, Base de données du Muséum National d'Histoire Naturelle, [http:// www.somali.asso.fr/clemam/index.php], consulté le 28 décembre 2013.

DAIre M.-Y., 2003 - Le sel des Gaulois, Éditions Errance, 150 p.

Dupont C., 2006 - La malacofaune des sites mésolithiques et néolithiques de la façade atlantique de la France. Contribution à l'économie et à l'identité culturelle des groupes concernés, Oxford, BAR International Series 1571, 439 p.

Dupont C. et Gruet Y., 200 I - «Au Néolithique dans le centreouest de la France, la pêche des coquillages reflète-t-elle l'environnement marin? ", in L'HelgouACH J. et BRIARD J. (dir.), Systèmes fluviaux, estuaires et implantations humaines de la préhistoire aux grandes invasions, Actes des congrès nationaux des sociétés historiques et scientifiques. $124^{\mathrm{e}}$ Nantes, 1999 ,

2. Une étude est actuellement en cours dans le cadre du PCR Bronze à propos des céramiques des sites du Pontreau et la Roussellerie-I'Ermitage par Yann Viau, Quentin Favrel, Christophe Maitay et Roland le Guevellou. Ils vont également reprendre la question des vases à sel sur ces sites (Favrel, 2014).

3. La céramique présente dans le foyer, vu sa technologie très proche des moules à sel du Néolithique récent, n’est pas une céramique de cuisson mais un moule à sel (macroporosité recherchée).
Éditions du Comité des travaux historiques et scientifiques. Paris, p. 183-199.

FAVREL Q., 20I4 - La transition du III au II millénaire au regard des productions céramiques du Campaniforme et $d u$ Bronze ancien en Bretagne et Pays de la Loire, Mémoire de Master 2, Université de Nantes, 2 vol., 698 p.

Gomez de Soto J., 2013 - «En France de l'Ouest, de la Loire à l'Aquitaine septentrionale, de la céramique cannelée au style RSFO. Diffusion ou polygénie? ", in LeCLERQ W. et Warmenbol E., Échanges de bons procédés : la céramique du Bronze final dans le nord-ouest de l'Europe, Actes du colloque international de l'université libre de Bruxelles, 1 et 2 octobre 2010, Bruxelles, CreA-Patrimoine, p. 267-285.

Joussaume R. et Pautreau J.-P., I990 - La Préhistoire du Poitou, Rennes, Éditions Ouest-France Université, 599 p.

Mougne C., en préparation - Exploitation et utilisation des invertébrés marins durant la protohistoire dans l'Ouest de la France, Doctorat, Université de Rennes 1.

Petrequin P. et Weller O., 2007 - « $\mathrm{Xv}^{\mathrm{e}}$ siècle av. J.-C. : la reprise de la croissance démographique dans le Jura ", in Mordant C., Richard H. et Magny M. (éd.), Environnements et cultures à l'âge du Bronze en Europe occidentale, Actes du $129^{\mathrm{e}}$ congrès CTHS, Besançon, 2004, Paris, éd. CTHS, coll. « Documents préhistoriques du CTHS ; 21 ", p. 197-210.

Poppe T. et Goto Y., I99I - European Seashells: Polyplacophora, Caudofoveata, Solenogastra, Gasteropoda. Germany, Verlag Christa Hemmen, vol. 1, 352 p.

Poppe T. et Gото Y., I 993 - European Seashells: Scaphopoda, Bivalvia, Cephalopoda. Germany, Verlag Christa Hemmen, Vol. 2, 221 p.

Reschreiter H. et Kowarik K., 2009 - " The earliest traces ", in Kern A., Kovarik K., Rausch A.W., Reschreiter H. (éd.), Kingdom of Salt. 7000 years of Hallstatt, Wien, Natural History Museum, VPA 3, p. 48-65.

Ters M., 1961 - La Vendée littorale, étude de géomorphologie, Rennes, 578 p.

Tessier M. et Bernard J., I995 - "La Roussellerie-L'Ermitage en Saint-Michel-Saint-Brévin (Loire-Atlantique), un site d'occupation continue du Néolithique final au Bronze final ", Bulletin de la Société préhistorique française, 1995, tome 92, 4, p. 479-498.

VIAU Y., 2005 - Le Pontreau 2, commune de Beauvoir-sur-Mer (Vendée), Rapport final d'opération, Inrap, SRA Pays-de-laLoire, $188 \mathrm{p}$.

Weller O., 2004 - «Produire du sel préhistorique : de la fonction des vestiges aux fonctions du produit ", in Bodu P. et Constantin C. (dir.), Approches fonctionnelles en préhistoire, Actes du XXV congrès préhistorique de France, 2000, Nanterre, SPF, p. 445-453. 
Zusammenfassung: Hinweise auf die Ausbeutung mariner Ressourcen am Ende des 2. Jahrtausends v. Chr.: die bronzezeitliche Feuerstelle bei Batardières (Saint-Vincent-sur-Jard, Vendée, Frankreich) - Im Rahmen wiederholter Flurbegehungen, die in der Folge des Orkans Xynthia durchgeführt wurden, konnten zwei archäologische Fundstellen (locus) oberhalb der Steilküste von Saint-Vincent-sur-Jard (Vendée, Frankreich) entdeckt werden. Wenn auch ihre räumliche Ausdehnung gering ist, so kann durch ihre Lage nahe der Abbruchkante der Steilküste ihre mögliche Zerstörung infolge extremer Wetterereignisse oder durch Hochwasser bei Sturmfluten nicht ausgeschlossen werden. Im Gegensatz dazu sind die Fundplätze durch Betreten derzeit nicht gefährdet, da sie gegenüber den Wanderern durch eine schützende Umzäunung abgegrenzt sind. Eine der Fundstellen umfasst die Reste einer Feuerstelle, die vermutlich als keramisches Fundgut die Überreste ein- und derselben Vase enthält. Die zugehörigen Scherben weisen zwei Besonderheiten auf: einerseits zeigen sie an, dass die Scherben in der Feuerstelle der Hitze ausgesetzt wurden, anderseits erlaubt ihr blasiges Erscheinungsbild die Hypothese, dass es sich um ein Gefäß zum Erhitzen von Salzlake gehandelt hat, mit dessen Hilfe Salzbrote erzeugt werden sollten. Diese Annahme konnte durch eine kleine Serie chemischer Analysen bestätigt werden. Im Randbereich der Feuerstelle spricht eine kleine Ansammlung von Muschelschalen dafür, dass wir eine Nutzung zur Speisebereitung annehmen müssen. Abschließend soll noch auf zwei Keramikscherben verwiesen werden, die im Randbereich der Feuerstelle gefunden wurden. Das Set ist mit dem Ende des zweiten Jahrtausend vor Christus datiert.

Resumen: Índices de explotación de los recursos marinos a finales del segundo milenio antes de Cristo: el hogar de la Edad de Bronce en Batardières (Saint-Vincent-sur-Jard, Vendée) - Durante prospecciones pedestres repetidas, después de la tormenta Xynthia, dos pequeños locus arqueológicos aparecieron en la cima del acantilado en Saint-Vincent-sur-Jard (Vendée). Si su influencia no es significativa, su ubicación en frente del acantilado les hace débiles en frente a la destrucción por fenómenos meteorológicos o por el ataque desde el mar durante las mareas más altas. Ellos no temen, por contra, el pisoteo ya que el paso de los caminantes se canaliza por una valla protectora que separa los restos. Uno de los locus contiene los restos de un hogar que contiene elementos probablemente originarios de un mismo vaso. Los fragmentos que lo componen tienen dos características: la primera muestra que se calentaron en la estructura de la combustión, la segunda por su aspecto vacuolar, permite considerar la hipótesis de un recipiente para calentar la salmuera con el fin de obtener un pan de sal, hipótesis confirmada por una pequeña serie de análisis químicas. En los afueras de la casa, una gran cantidad de conchas también indica una función de cocción. Por último, otros dos restos cerámicos fueron encontrados cerca de la casa. El conjundo se fecha al final del segundo milenio antes de Cristo.

Schlüsselwörter: Bronzezeit, Küstenlinie, Feuerstelle, Keramikgefäße, Muscheln, Atlantikküste, Vendée.

Palabras clave: Edad de Bronce, costa, hogar, cerámicas, conchas, costa atlántica, Vendée. 